\title{
A Pilot Study to Evaluate the Efficacy of IZN 6D4 for the Treatment of Diabetic Foot Ulcers
}

\section{Menachem Oberbaum and Jonathan Rosenblum*}

Diabetic Foot Service, Department of Orthopedics, Shaarei Zedek Medical Center, Jerusalem, Israel

*Corresponding author: Jonathan Rosenblum, Director, Diabetic Foot Service, Department of Orthopedics, Shaarei Zedek Medical Center, Israel, Tel: +972-50-595-5161; E-mail: diabfootman@gmail.com

Received date: May 24, 2017; Accepted date: June 22, 2017; Published date: June 29, 2017

Copyright: (c) 2017 Oberbaum M, et al. This is an open-access article distributed under the terms of the Creative Commons Attribution License, which permits unrestricted use, distribution, and reproduction in any medium, provided the original author and source are credited.

\begin{abstract}
Diabetic foot ulcers (DFU), which can affect up to $15 \%$ of individuals with diabetes, remain a major unmet medical need. Left untreated, DFU seriously impact on quality of life and level of function. Conventional treatment is costly and often ineffective. In severe cases, patients with DFU may undergo painful amputations. IZN 6D4 is an impregnated hydrogel containing active pharmaceutical ingredients that have both wound-healing promoting and anti-inflammatory activities This is an observational human clinical trial study involving 15 patients treated with IZN 6D4. Subjects in the study had DFU which were categorized as being at stage 1A or greater using the University of Texas Health Science Center, San Antonio scale (UTHSC) grading system. Treatment lasted 21 days, and during the course of the study, patches were applied every 3-4 days. Response to therapy was measured by extent and depth of the lesion (wound grid and digital photography), qualitative assessment of wound healing (tissue granulation), and the rate of wound closure over the trial period. Results of the study showed that $40 \%$ of subjects had $75 \%$ closure of the wound and $27 \%$ had complete wound closure. Nearly all patients had evidence of active wound healing (e.g., presence of granulation tissue), and percent mean wound reduction for the entire study group was $67.7 \pm 26.4 \%$. Photo documentation of wound healing showed impressive differences between pre- and posttreatment appearances of diabetic ulcers in many of the patients studied. Based on the promising data from preclinical studies and the observational clinical trial provide strong justification for initiating a large-scale, longer duration clinical trial evaluating the efficacy of IZN 6D4 hydrogel in the treatment of patients with DFU.
\end{abstract}

Keywords: Diabetic foot ulcers; Pilot Study; Clinical trial; Granulation

\section{Introduction}

The treatment of diabetic foot ulcers remains a major unmet medical need [1]. DFU has an incidence rate of $15 \%$ among all diabetic patients, and is more common in those with long-standing diabetes and poor glycemic control [2]. Conservative management of DFU is frequently ineffective and between $14-20 \%$ of diabetics with foot ulcers will eventually require amputation [3]. DFU are the most common cause of non-traumatic amputation, and the cumulative 10-year incidence of lower-extremity amputation is $5 \%$ for diabetics diagnosed before the age of 30 and $7 \%$ among those with later onset of diabetes ( $>30 \mathrm{y})$. The morbidity and mortality of diabetic foot ulcers are high as evidenced by the prolonged hospitalization of patients with foot ulcers, and the bleak $27 \%$ 5-year survival rate after amputation is worse than for most cancers [4].

The economic burden of DFU for society is significant with a recent study finding that the cost of caring for diabetic ulcers ranged from $\$ 1,892$ to $\$ 27,721$ per episode based on severity with the average costs being $\$ 13,179$ per episode [5]. Amputation costs are estimated to be $\$ 6$ billion a year in the US [2] and the total cost of care for DFU in 2001 was $\$ 10.9$ billion [6]. It has been estimated that up to $20 \%$ of the annual costs of caring for diabetes is directly or indirectly (i.e., loss of productivity) related to DFU [6]. Beyond, the financial costs, there is a significant human toll related to DFU in terms of loss of mobility and reduced quality of life [7]. Development of more effective treatment modalities for DFU would significantly reduce the financial and human toll, and benefit both individual patients and society at large.

A significant amount of research has been devoted to determine the specific etiology of DFU and to answer the question of why there is impaired healing of skin ulcers in diabetes $[1,8,9]$. The impaired healing may result from numerous factors including changes in growth factor milieu, impaired fibroblast function, decreased immunologic function, and defects in the extracellular matrix (ECM) environment in the region of the wound.

In diabetes, several factors, both external and internal, work to retard healing. These include neuropathy, vascular insufficiency, microangiopathy, repeat trauma and increased susceptibility to infection. Microangiopathy is particularly important as the thickened basement membrane surrounding the ulcer impairs inflammatory cell recruitment affecting the first and second stages of wound healing [10]. At the cellular level, fibroblasts in the diabetic ulcer show signs of senescence and have a decreased proliferative response to growth factors as compared to fibroblasts in non-diabetic wounds [11,12]. At the molecular level, biopsies taken from diabetic ulcers have lower levels of TGF- $\beta$ and type 2 TGF- $\beta$ receptors as compared to wounds from non-diabetic individuals $[13,14]$. This is a critical factor as TGF mediated signal transduction is important in wound healing [15]. DFU, similar to other poorly healing wounds, have elevated levels of metalloproteinases (MMP) that destroy growth factors, surface receptors of cells involved in wound healing, and ECM proteins that are necessary for healing [16,17]. In addition, there is a reduction of tissue inhibitor of MMP which inhibits the activity of destructive metalloproteinases [18]. 
Page 2 of 4

IZN 6D4 is a hydrogel containing naturally derived pharmaceutical ingredients designed to improve wound healing in diabetics with DFU who have elevated levels of metalloproteinases in their foot ulcers. It was found that IZN 6D4 had an impact on growth factors and extracellular matrix factors that are found in wounds. A key finding was that in inflamed biopsy tissue obtained from subjects treated with the active ingredient, there was a significant decrease in the levels of metalloproteinases.

\section{Materials and Methods}

This is an observational study to determine the efficacy of a hydrogel impregnated with IZN 6D4 in treating DFU. The active product ingredients of the hydrogel consisted of IZN 6D4 $1.5 \mathrm{~g}$ mixed with 15 gm of hydrogel. The gel mixture was applied by the study physician as a $2 \mathrm{~mm}$ thick layer covering the entire wound bed. The gel was subsequently covered by a sterile saline-soaked gauze and fixed in place.

Fifteen patients with DFU were enrolled. To qualify for enrollment, patients had to have confirmed diabetes, be between the ages of 18-75 years, and have foot ulcers categorized as $1 \mathrm{~A}$ or greater based on the University of Texas Health Science Center, San Antonio scale (UTHSC). Exclusion criteria including sensitivity to any of the ingredients in the hydrogel or the API, inability to apply the API preparation to the wound site, pregnancy or the desire to become pregnant, and moderate to severe peripheral vascular disease. The duration of treatment with the hydrogel was 21 days. On the first day of the study, the patient was enrolled and baseline statistics were obtained including the location of the ulcer, measurements of the depth and width of the lesion, and the UTHSC classification. The wound was debrided according to standard procedures, and the test substance was applied and covered with a saline dressing. Subsequently, patients returned every 3-4 days for assessment by the physician, further debridement, and application of a new patch. A comprehensive assessment was done on day 21, and when possible, patients were followed for up to a period of 12 weeks.

Several methods were used to evaluate the wound. The depth of the wound was measured with a standard calibrated probe. Surface area of the wound was assessed using a wound grid (Convatec). Digital photographs (three photographs at magnifications of $1 x, 2 x, 3 x$ of increasing magnification) at a fixed distance from the wound were obtained. Qualitative assessment of tissue content and quality of healing was done by the clinical investigator who visually estimated the percentage of wound area consisting of each of the following tissue types: necrotic, fibrotic, granulation, and epithelialized. Data was recorded on case report forms for each patient and subsequently entered in a Microsoft Excel spreadsheet.

Additional data was obtained regarding adverse events that could be potentially related to the test drug. Compliance was assessed by monitoring attendance during the scheduled visits. Patients who failed to maintain their scheduled visits were released from the study.

There were several outcome variables for this observational study that were assessed at 21 days and included the number of patients with complete healing, percent change in either surface area or depth of wound during the course of the study, changes in UTSHC grade, the velocity of healing in terms of reduction of wound surface area and depth over time, and subjective evaluation of wound healing based on granulation tissue. For long-term follow-up, the main outcome variable was closure. An intention to treat standard was utilized in the sense that patients lost to follow-up before complete healing were considered as treatment failures.

\section{Results}

The enrollment goal of the study was achieved and 15 sequential volunteers were enrolled after they signed informed consent. A complete data set for the 15 patients was obtained at 3 weeks. No SAEs were recorded related to the medication. Treatment with IZN 6D4 was well tolerated among all the patients regardless of extent or severity of their ulcers.

The demographic and clinical aspects of the patient population are shown in Table 1 . The population was elderly with a preponderance of males. A third of the patients had either chronic or recurrent DFU $(>6$ months). A majority of the DFU were neuropathic in type (11 patients had wounds classified as non-ischemic) according to UTHSC (classified as A). Most of the ulcers were superficial with only 4 patients having ulcers that reached to the tendon or capsule (stage 2 based on UTHSC).

\begin{tabular}{|l|l|}
\hline Age $(\mathrm{y})$ & $63.6 \pm 10.9$ \\
\hline M:F & 1.5 \\
\hline Duration before treatment & \\
\hline$<6$ months & 10 \\
\hline$\geq 6$ months & $5(6$ mo-4 y) \\
\hline UTHSC score (distribution) & \\
\hline IA & 9 \\
\hline IB & 1 \\
\hline IC & 2 \\
\hline IIA & 2 \\
\hline IIC & 2 \\
\hline
\end{tabular}

Table 1: Clinical characteristics of subjects.

The results of treatment with IZN 6D4 combined with conventional treatment for three weeks are summarized in Table 2. All subjects responded to treatment in terms of reduction of the size of their ulcers. The mean surface area of the ulcer decreased from $6.08 \pm 6.54$ to $3.01 \pm$ $5.08 \mathrm{~cm}^{2}$ ( $\mathrm{p}<0.0001, \mathrm{t}$ test for two-tailed paired samples). In terms of wound closure, $27 \%$ had complete closure during the three weeks, while $40 \%$ of the subjects had $75 \%$ or greater closure

\begin{tabular}{|l|l|}
\hline Initial area $\left(\mathbf{c m}^{2}\right)$ & \\
\hline Mean & $6.08 \pm 6.54$ \\
\hline Median & 3.92 \\
\hline Range & $1.08-25.95$ \\
\hline Final area $\left(\mathbf{c m}^{2}\right)$ & \\
\hline Mean & $3.01 \pm 5.08$ \\
\hline Median & 0.98 \\
\hline Range & $0.0-18.90$ \\
\hline
\end{tabular}




\begin{tabular}{|l|l|}
\hline Response & \\
\hline $100 \%$ closure & $4 / 15(27 \%)$ \\
\hline $75 \%$ closure & $6 / 15(40 \%)$ \\
\hline Percent wound reduction & \\
\hline Mean & $67.7 \pm 26.4$ \\
\hline Median & 69.8 \\
\hline Range & $19-100$ \\
\hline $100 \%$ granulation & $14 / 15$ \\
\hline$\%$ granulation (mean) & $99.0 \pm 3.87$ \\
\hline
\end{tabular}

Table 2: Response to treatment with IZN 6D4.

Since initial wound size varies, percent wound reduction was also analyzed. Again, the response was varied (Figure 1) but the average reduction of ulcer size was $67.7 \%$. Except for one patient, most patients had an immediate response during the first week of treatment. In the third week of the trial, there was a plateau effect seen in four of the patients (Figure 1).

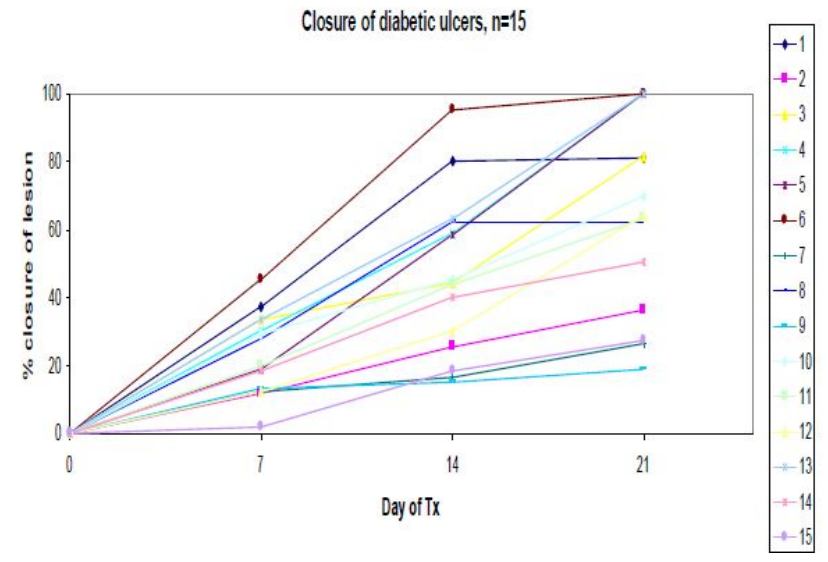

Figure 1: Plateau effect of diabetic ulcers.

Of note is that although the percent closure over three weeks was greater for individuals with ulcers less than 6 months, this difference was not statistically significant $(74.9 \%$ with DFU $<6$ months vs. $53.4 \%$ with DFU $\geq 6$ months; $\mathrm{p}=0.14$, two-tailed $\mathrm{t}$ test). Another indication of the efficacy of IZN 6D4 was the degree of granulation. Granulation is an important stage in the healing process, and all 15 patients had complete granulation of their wounds. The impact of granulation is also demonstrated by the fact that all 4 patients with deep ulcers classified as Stage 2 in the UTSHC classification reverted to Stage 1. Figure 2 shows visual evidence in two cases of the degree of healing both in terms of granulation tissue and degree of closure.

After cessation of treatment at 3 weeks, the patients were followed for an additional period that ranged up till 12 weeks. Using closure as an endpoint, 9 out of $15(60 \%)$ responded to IZN 6D4 (Figure 2).

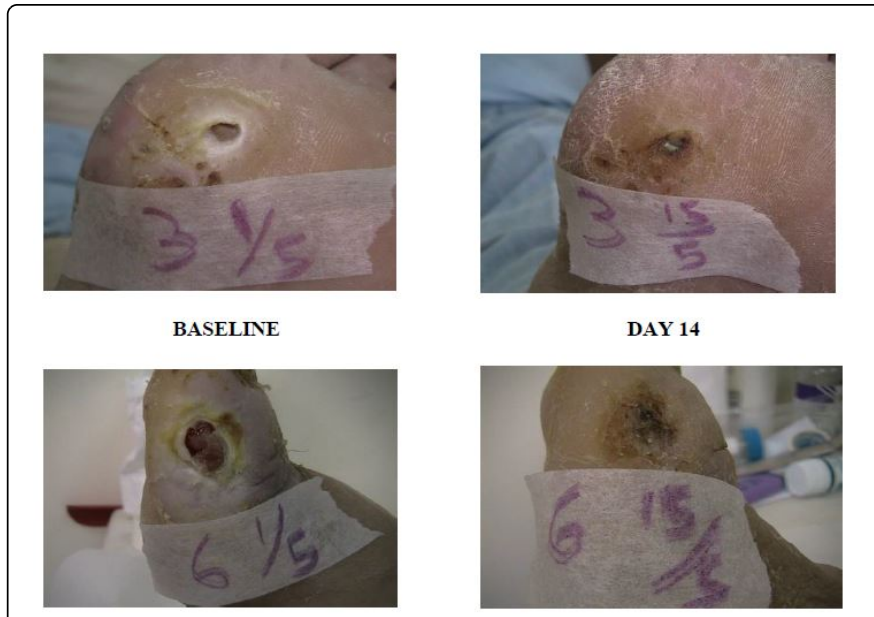

Figure 2: Visual evidence of healing both in terms of granulation tissue and degree of clouser.

Of the 6 who were considered treatment failures based on the intention to treat protocol, one patient was non-compliant with treatment, two were lost to follow-up during the third week of the study, and the other 3 were lost to follow-up during the next 9 weeks.

\section{Discussion}

The preclinical studies presented above provide evidence that IZN $6 \mathrm{D} 4$, a polymolecular API, can facilitate wound healing by modulating the molecular environment of the wound thereby increasing the rate of wound closure

Using the wound closure model, the effects of IZN 6D4 on metalloproteinases, TGF- $\beta$, and healing-associated cytokines were assessed. IZN 6D4 significantly inhibited fibroblast MMP-2 production as measured over a 24 hour period by zymography, a test of functional enzymatic degradation of specific substrates. As mentioned above, the reduction in MMP-2 is an important finding as this MMP is elevated in DFU wounds as compared to non-diabetic wounds.

Healing-associated cytokines such as IL- 1 and IL- 6 are important in wound healing [19], and IL-6 is a critical factor for fibroblast proliferation [20]. In cultured fibroblasts, IZN 6D4 induced secretion of IL-6, and was synergistic with IL-1 in raising levels of IL-6. IZN 6D4 also stimulated the secretion of collagen by fibroblasts.

The limitation of this study was the lack of a control group of patients receiving conventional therapy alone. It is possible that the improvement seen in the ulcers was solely related to conventional therapy. However, when comparing the outcome obtained with three weeks of IZN 6D4 therapy to the natural history of DFU patients receiving conventional therapy, the results are very promising.

For example, in one study of 449 subjects in which the patient population was similar to that of the present study, 55\% percent had complete healing after 6 months with a median time to healing of 78 days. 21 In our study, 4 patients had healing by 3 weeks and another 5 had complete healing by 12 weeks for a combined percentage of $60 \%$ suggesting that the rate of healing was accelerated when IZN 6D4 was utilized. 
Our results also compare favorably to that of Promogran and Apligraf. Promogran, a wound dressing consisting of collagen and oxidized regenerated cellulose which is a protease modulating matrix that promotes healing. In a multicenter trial with consisting of 276 patients receiving Promogran or standard care, the complete closure rate after 12 weeks of treatment was $37 \%$ versus $28.3 \%$ in the control group $(p=0.12)[21,22]$. This is in comparison to our study in which there was a projected complete closure rate of $60 \%$ after 12 weeks. IZN 6D4 was equivalent or better than Apligraf, a bioengineered skin substitute impregnated with keratinocytes and fibroblasts. In a recently published twelve week study using Apligraf together with a non-weight bearing regimen, a closure rate of $51.5 \%$ was obtained for Apligraf [23]. This was significantly better than the control group which received conventional care and a non-weight bearing regimen for which the response rate was $26.3 \%(p=0.049)$. When IZN $6 D 4$ is compared to results from the Apligraf study, it can be demonstrated that the response to IZN $6 \mathrm{D} 4$ is more rapid, and the response rate is greater even though a formal non-weight bearing regimen was not instituted in the IZN 6D4 study. IZN 6D4 also performs well when compared to other biological-based wound coverings including Dermagraft, OASIS wound matrix, and Regranex gel [24,25]. Extrapolating from the individual subject data, an additional 4 patients were on a trajectory for completing healing by 4-6 weeks. Similar to other studies [22], patients with ulcers of less than 6 months duration had a better total closure response (40\%) as all four patients who had a complete response had ulcers of less than 6 months duration.

What is most impressive about IZN 6D4 is the relatively short (3 week) duration of treatment. It could be expected that with a longer duration of treatment, the number of patients with a positive response would increase. Taking both the positive data from the pre-clinical studies and the results of the observational clinical trial, there is a strong justification for initiating a large-scale, longer duration placebo controlled clinical trial to evaluate the efficacy of IZN 6D4 hydrogel in the treatment of patients with DFU.

\section{References}

1. Sweitzer SM, Fann SA, Borg TK, Baynes JW, Yost MJ (2006) What is the future of diabetic wound care?. Diabetes Educ 32: 197-210.

2. Ramsey SD, Newton K, Blough D (1999) Incidence, outcomes, and cost of foot ulcers in patients with diabetes. Diabetes Care 22: 382-387.

3. Eldor R, Raz I, Ben YA, Boulton AJ (2004) New and experimental approaches to treatment of diabetic foot ulcers: a comprehensive review of emerging treatment strategies. Diabet Med 21: 1161-1173.

4. Reiber GE (2001) Epidemiology of foot ulcers. In: Bowker JH, Pfeffer F (editors). The Diabetic Foot.St. Louis, MO: Mosby pp: 13-32.

5. Stockl K, Vanderplas A, Tafesse E, Chang E (2004) Costs of lowerextremity ulcers among patients with diabetes. Diabetes Care 27: 2129-2134.

6. Boulton AJ, Vileikyte L, Ragnarson-Tennvall G, Apelqvist J (2005) The global burden of diabetic foot disease. Lancet 2005 366: 1719-1724.

7. Ribu L, Hanestad BR, Moum T, Birkeland K, Rustoen T (2007) A comparison of the health-related quality of life in patients with diabetic foot ulcers, with a diabetes group and a non diabetes group from the general population. Qual Life Res $16: 179-189$.
8. Mustoe TA, O'Shaughnessy K, Kloeters O (2006) Chronic wound pathogenesis and current treatment strategies: a unifying hypothesis. Plast Reconstr Surg 117: 35S-41S.

9. Falanga V (2005) Wound healing and its impairment in the diabetic foot. Lancet 366: 1736-1743.

10. Ngo BT, Hayes KD, DiMiao DJ, Srinivasan SK, Huerter CJ, et al. (2005) Manifestations of cutaneous diabetic microangiopathy. Am J Clin Dermatol 6: 225-237.

11. Loots MA, Lamme EN, Mekkes JR, Bos JD, Middelkoop E (1999) Cultured fibroblasts from chronic diabetic wounds on the lower extremity (non-insulin-dependent diabetes mellitus) show disturbed proliferation. Arch Dermatol Res 291: 93-99.

12. Loot MA, Kenter SB, Au FL (2002) Fibroblasts derived from chronic diabetic ulcers differ in their response to stimulation with EGF, IGF-I, bFGF and PDGF-AB compared to controls. Eur J Cell Biol 81: 153-160.

13. Galkowska H, Wojewodzka U, Olszewski WL (2006) Chemokines, cytokines, and growth factors in keratinocytes and dermal endothelial cells in the margin of chronic diabetic foot ulcers. Wound Repair Regen 14: 558-565.

14. Jude EB, Blakytny R, Bulmer J, Boulton AJ, Ferguson MW (2002) Transforming growth factor-beta 1, 2, 3 and receptor type I and II in diabetic foot ulcers. Diabet Med 19: 440-447.

15. Faler BJ, Macsata RA, Plummer D, Mishra L, Sidawy AN (2006) Transforming growth factor-beta and wound healing. Perspect Vasc Surg Endovasc Ther 18: 55-62.

16. Lobmann R, Schultz G, Lehnert H (2005) Proteases and the diabetic foot syndrome: mechanisms and therapeutic implications. Diabetes Care 28: 461-471.

17. Lobmann R, Zemlin C, Motzkau M, Reschke K, Lehnert H (2006) Expression of matrix metalloproteinases and growth factors in diabetic foot wounds treated with a protease absorbent dressing. J Diabetes Complications 20: 329-335.

18. Ladwig GP, Robson MC, Liu R, Kuhn MA, Muir DF, et al. (2002) Ratios of activated matrix metalloproteinase- 9 to tissue inhibitor of matrix metalloproteinase- 1 in wound fluids are inversely correlated with healing of pressure ulcers. Wound Repair Regen 10: 26-37.

19. Efron PA, Moldawer LL (2004) Cytokines and wound healing: the role of cytokine and anticytokine therapy in the repair response. J Burn Care Rehabil 25: 149-160.

20. Mateo RB, Reichner JS, Albina JE (1994) Interleukin-6 activity in wounds. Am J Physiol 266: R1840-R1844.

21. Jeffcoate WJ, Chipchase SY, Ince P, Game FL (2006) Assessing the outcome of the management of diabetic foot ulcers using ulcer-related and person-related measures. Diabetes Care29: 1784-1787.

22. Veves A, Sheehan P, Pham HT (2002) A randomized controlled trial of Promogran (a collagen/oxidized regenerated cellulose dressing) vs standard treatment in the management of diabetic foot ulcers. Arch Surg 137: 822-827.

23. Edmonds M (2009) Apligraf in the Treatment of Neuropathic Diabetic Foot Ulcers. Int J Low Extrem Wounds 8: 11-18.

24. Marston WA, Hanft J, Norwood P, Pollak R (2003) The efficacy and safety of Dermagraft in improving the healing of chronic diabetic foot ulcers: results of a prospective randomized trial. Diabetes Care 26: 1701-1705.

25. Niezgoda JA, Van Gils CC, Frykberg RG, Hodde JP (2005) Randomized clinical trial comparing OASIS Wound Matrix to Regranex Gel for diabetic ulcers. Adv Skin Wound Care 18: 258-266. 